\title{
Micro Mixer with Fast Diffusion
}

\author{
Ryo Miyake ${ }^{1}$, Theo S.J. Lammerink ${ }^{2}$, Miko Elwenspoek ${ }^{2}$ \\ Jan H.J. Fluitman 2 \\ 1. Mechanical Engineering Research Laboratory, Hitachi, Ltd. \\ 502, Kandatsu, Tsuchiura 300 Japan \\ 2. MESA Research Institute, University of Twente, \\ P.O. Box 217, 7500 AE Enschede, The Netherlands
}

\begin{abstract}
A new concept for micro-mixing of liquid is introduced and feasibility is demonstrated. The mixer allows fast mixing of small amounts of two liquids and it is applicable to micro-liquid handling systems [1]. The mixer has a channel for the liquid, an inlet port for the reagent, and a $2.2 \mathrm{~mm} \times 2 \mathrm{~mm} \times 330 \mu \mathrm{m}$ mixing area, and its bottom has 400 micro-nozzles $(15 \mu \mathrm{m} \times 15 \mu \mathrm{m})$. Through these nozzles, a reagent is injected into the sample liquid, making many micro-plumes. These plumes increase the contact surface between the two liquids drastically, and hasten the speed of the mixing by diftusion. The nozzle holes and channel are etched using $\mathrm{KOH}$ into a 3-inch (100)-Si wafer from both sides. The fabrication process is extremely simple. Flow visualization by microscope shows the mixing is complete within a few seconds, and absorptiometry using a pair of optical fibers showed that a homogeneous state of mixing is reached in 1.2 seconds, when the total volume injected is $0.5 \mu \mathrm{l}$ and the injection flow rate is $0.75 \mu \mathrm{l} / \mathrm{s}$.
\end{abstract}

\section{INTRODUCTION}

Various sorts of micro mechanical devices have been developed using micro-technologies. Micro-liquid handling devices such as micropumps [2], active [3] and passive [4] microvalves has received considerable attension, and current studies are trying to integrate them as micro-liquid handling systems [1].

The basic concept of micro-liquid handling systems has been proposed as illustrated in Fig. 1. It consists of micro-liquid dosing systems, a micro-mixer and reactor, a detector, and micro-channels connecting the devices. A sample liquid is introduced into the micro mixer by the dosing system, mixed with a reagent and measured by the detector. Compared to an ordinary automatic chemical analyzer, this system has the advantages of superior portability, high accuracy, low consumption of reagents, and the ability to handle small amounts of sample [5].

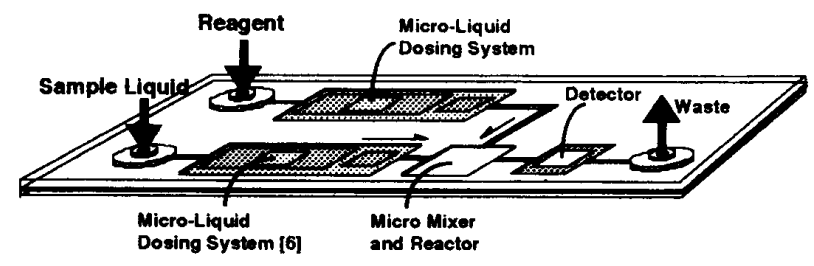

Fig. 1 Basic Concept of Micro-Liquid Handling System [1]
A micro-liquid dosing system with micropump and flowmeter has been presented [6], which is able to transport a small amount $(\sim 1 \mu \mathrm{l})$ of liquid accurately at a flow rate of less than $1 \mu \mathrm{l} / \mathrm{s}$. Microliquid handling systems require micro mixers that can be easily integrated with this dosing system on a single chip. The properties necessary for the micro mixer are

1) the ability to mix small amounts of liquids $(\sim 1 \mu$ )

2) a simple method for mixing quickly and uniformly

3) a simple fabrication process for integration on a single chip

In general, mixing is performed by a combination of convection and molecular diffusion. However, when small amount of liquids are mixed, the Reynolds number is very small, and it is, therefore, difficult to generate sufficient convection for the mixing.

We propose a new concept for mixing liquids when the Reynolds number is small, and demonstrate a mixer which allows fast mixing of small amounts of two liquids, which is also applicable in micro-llquid handling systems.

\section{PRINCIPLE}

Molecular diffusion plays a more important role in a flow with a small Reynolds number, since it is driven by a concentration difference ( Fick's law ). First, we consider the characteristics of molecular diffusion in the micro-domain, and utilize it to create a micro mixer.

Diffusion speed increases with the rise of the contact surface of two liquids. In an ordinary mixing chamber, the turbulence flow by stirrer provides minute segregated domains of liquid, which increase the contact surface [7]. For quick diffusion, it is required to create this segregated state.

The time taken when molecules travel is increasing in proportion to the square value of the distance [8]. For instance, if the distance is $10 \mu \mathrm{m}$, the traveling time in water is around $1 \mathrm{sec}$., and if $1 \mathrm{~mm}$, the time is on the order of $1000 \mathrm{sec}$. Therefore, the distance for diffusion should be short enough to reduce the traveling time.

The basic idea of the micro mixer, reflecting the above 2 characteristics, is illustrated in Fig. 2. The mixer has an area for mixing which is very flat and thin with many micro-nozzles on the bottom. During operation, first, the mixing area is filled with one liquid, and the other liquid is injected into the area through the many micro nozzles, making many micro-plumes. These plumes increase the contact surface. The nozzles are positioned very closely in rows, 10-100 $\mu \mathrm{m}$ apart, in order that the plumes may quickly diffuse for this distance. Thus, effective mixing will be performed without any additional driving.
0-7803-0957-2/93 $\$ 3.00$ @ 1993 IEEE 


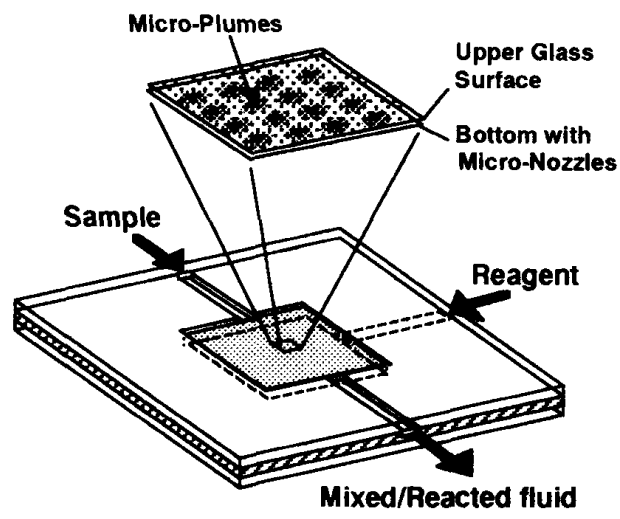

Fig.2 Basic idea of Micro-Mixer

\section{Feasibility test}

Numerical analysis of the diffusion flow was employed to check the feasibility of the concept. Figure 3 is a comparison of diffusion efficiency between a large single plume (a) and in the many micro-plumes from the 400 micro-nozzles (b). The former case (a) may be considered as a simple junction of two liquids. Here it should be noted that the latter case (b) was simulated for only one plume, since the flow pattern from each nozzle is similar. The diffusion coefficient $D=1.0 \times 10^{-3} \mathrm{~mm}^{2} / \mathrm{s}$ was used to represent molecular diffusion in water. Please note that this value of $D$ is used for all calculations in this paper. The parameter for estimating the degree of mixing is given by a percentage of the total mass injected where concentration decreases less than $50 \%$.

$$
\begin{aligned}
& \text { Degree of Mixing }=\frac{\int_{v} c_{d} d v}{\int_{v} c d v} \times 100(\%) \\
& c_{d}=c \leq 0.5
\end{aligned}
$$

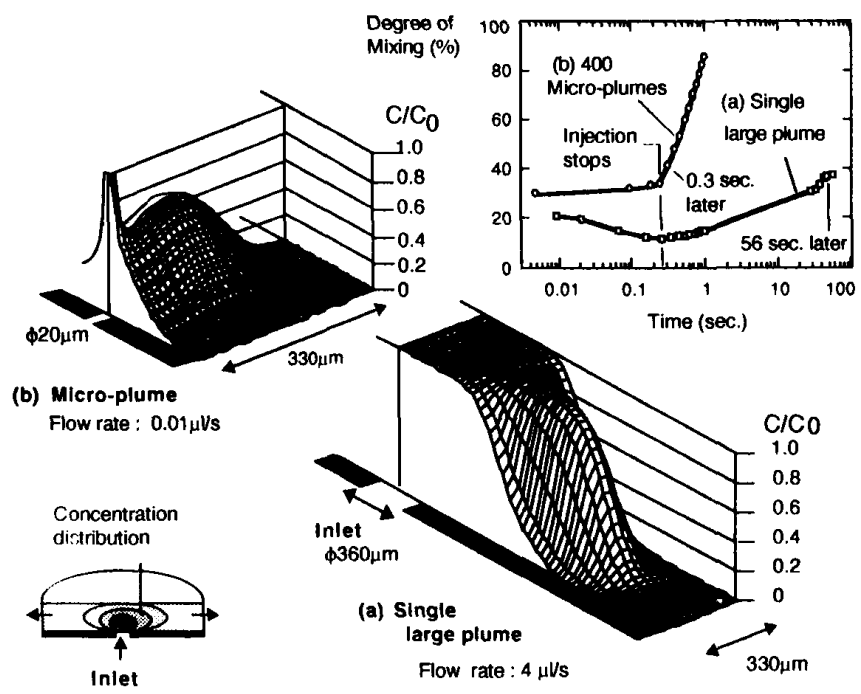

Fig.3 Feasibility Test of Micro Mixer
Here, the value of $50 \%$ is used as an index of the degree of mixing. $c$ and $c_{d}$ is standardized by the initial concentration co. The total liquid injected is $1 \mu \mathrm{l}$, and the flow rate is $4 \mu \mathrm{l} / \mathrm{s}$. Therefore, it stops at $0.25 \mathrm{sec}$. This result indicates that mixing in the micromixer is 100 times faster than in case (a), comparing the time to reach a value of $40 \%$. A 3-D diagram of concentration distribution indicates that all portions of the micro-plume is diffused in $1.0 \mathrm{sec}$. Hence, this analysis predicts that this mixer has a property to finish mixing within a few seconds.

\section{DESIGN OF MICRO MIXER}

The main parameters for designing a micro mixer are the number of micro-nozzles and the distance between each nozzle, which determines the area of mixer, the height of the mixing area (h), the size of the micro-nozzles (d), and the injection flow rate of the micro-plumes (q).

The distance between each nozzle is set to be $100 \mu \mathrm{m}$ due to the fabrication process ; (orientation dependent etching with $\mathrm{KOH}$ solution). The area for mixing was determined to be $2 \mathrm{~mm} \times 2$ $\mathrm{mm}$, which provides enough volume to mix a microliter of liquid, and allows space for $\mathbf{4 0 0}$ micro-nozzles.

Height (h), size of nozzle (d), and injection flow rate $(q)$ are discussed using numerical analysis, since they are directly related to the diffusion flow. A finite differential method is used to simulate changes in the micro-plume over time [9]. Figure 4 depicts the model of the flow passage, which is symmetrical around the axis of the micro-nozzle in order to simplify to a 2-dimensional analysis (see Appendix). Here, a flow with a micro-plume comes out through the micro-nozzle and expands radially.

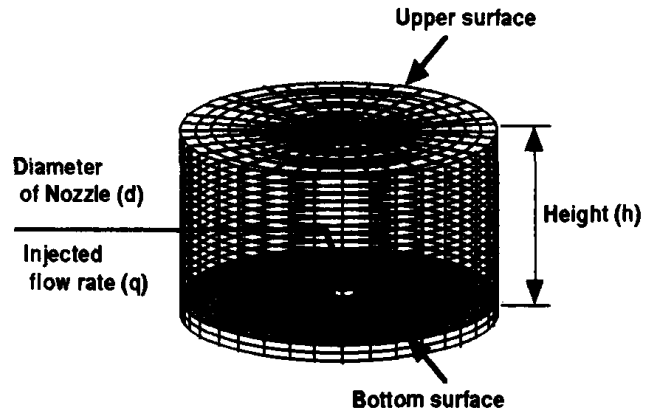

Fig. 4 Model of Flow Passage

The diffusion flow is well estimated using the center of gravity $\mathrm{zg}$ and the variance of mass diffused $\sigma[10]$

$$
\begin{array}{r}
z_{g}(t)=\frac{\int_{v} z(v) \cdot c(t, v) d v}{\int_{v} c(t, v) d v} \\
\sigma(t)=\frac{\int_{v}\left\{z(v)-z_{g}(t)\right\}^{2} \cdot c(t, v) d v}{\int_{v} c(t, v) d v}
\end{array}
$$




\section{EXPERIMENTAL RESULTS}

\section{Flow visualization}

Flow visualization is essential for a qualitative analysis of mixing process. Figure 10 shows the experimental setup, with a video camera to catch the quick change in the micro-plumes. $A$ high-precision syringe pump driven by a pulse motor controls small amounts of liquid accurately, and its flow rate can be varied from $0.01 \mu \mathrm{l} / \mathrm{s}$ to $0.8 \mu \mathrm{l} / \mathrm{s}$. A water supplier driven by pressurized air was used to flush the fluid inside the mixer. Instead of a reagent, a fluorescent dye, uranine; $\left(\mathrm{C}_{20} \mathrm{H}_{10} \mathrm{O}_{5} \mathrm{Na}_{2}\right)$ was used because it is easily visible even at low concentrations [11]

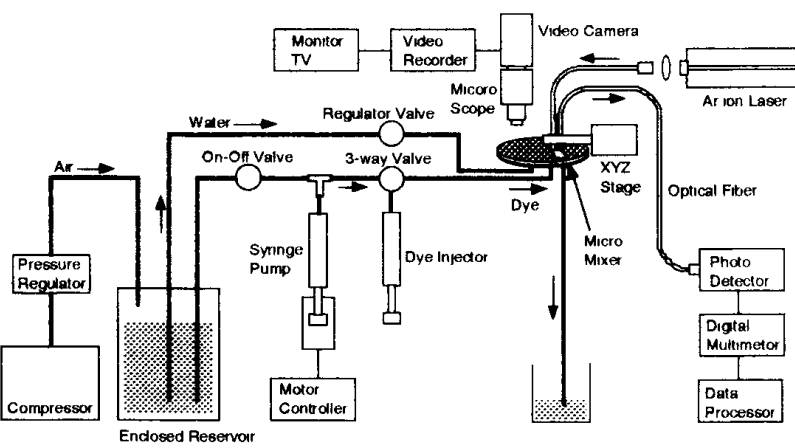

Fig. 10 Experimental Setup

Figure $11(\mathrm{a})$ is a microscopic view of the plumes, with a flow rate of $0.57 \mu \mathrm{l} / \mathrm{s}$ and a total of about $0.5 \mu \mathrm{l}$ of dye. It shows the dye diffuses into a homogeneous mixture within a few seconds, after the injection stops (at around $0.9 \mathrm{sec}$.), as the simulation models predict.

Figure 11 (b) shows the boundary between the mixing fluid and the water in contact with it, 1.5 minutes after dye injection. The boundary can still been seen clearly. This is because diffusion time is longer in proportion to the square of the distance as discussed already. This allows the mixing area to be treated as a micro-reaction beaker.

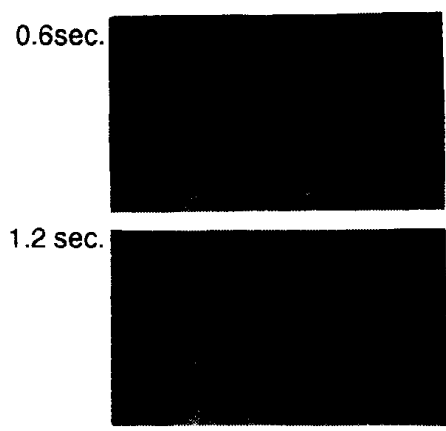

(a) Micro-Plumes

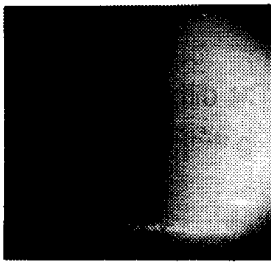

(b) Boundary of Mixing Area
Fig. 11 Microscopic View of Micro-Plumes

\section{Measuring method by absorptiometry}

The mixing process has also been observed using absorptiometry. Figure 12(a) shows the method and optical setup of absorptiometry for this mixer. Light from an optical fiber enters the mixing area by reflecting from the wall, and travels between the bottom and the upper reflector. At the other wall, the light re- flects upwards, and exits the area. Rhodamine $\left(\mathrm{C}_{28} \mathrm{H}_{30} \mathrm{~N}_{2} \mathrm{O}_{3} \mathrm{HCl}\right)$ is used as a dye, and an Argon ion laser $(514.5 \mathrm{~nm})$ is used for illumination as shown in Fig. 10, since the main absorption wavelength of Rhodamine is $520 \mathrm{~nm}$ [12]. The power of the light from the mixing area decreases with the absorption of dye. As shown in Fig. 10, the light is detected by a photo-detector through the other optical fiber.

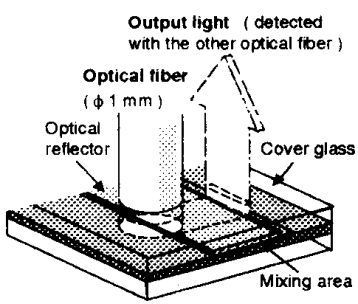

(a) Optical Setup for Absorptiometry

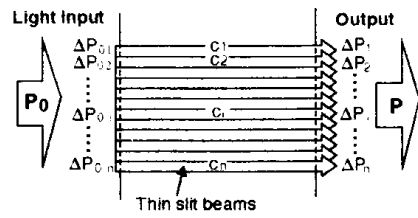

(b) Measuring Methoo
Fig. 12 Optical Setup for Measurement

The mixing area is very flat. Therefore, the light is considered as a integration of many thin slit beams, as in Figure 12(b). Each beam is absorbed along the beam path. The relation between initial optical power $\Delta \mathrm{P}_{0 i}$ and output optical power $\Delta \mathrm{P}_{\mathrm{i}}(\mathrm{t})$ for the $n$th beam is given by Lambert and Beer's law,

$$
\Delta P_{i}(t)=\Delta P_{0} \cdot \exp \left[-\varepsilon_{\lambda} \cdot c_{i}(t) \cdot 1\right]
$$

Here, $I$ is the length of beam path, and $c_{i}(t)$ is the mean concentration of dye along the beam path. $\varepsilon_{\lambda}$ is the absorption coefficient of wave lenght $l$. The integration from $i=1$ to $n$ gives the relation of total optical power between $P(t)$ and $P_{0}$ as follows:

$$
P(t)=\frac{P_{0}}{n} \sum_{i=1}^{n} \exp \left[-\varepsilon_{\lambda} \cdot c_{j}(t) \cdot l\right]
$$

After the injection stops, the dye disperses and becomes homogeneous at a time $t_{m}$, hence the relation between $P\left(t_{m}\right)$ and $P_{0}$ is as follows:

$$
P\left(t_{m}\right)=P_{0} \cdot \exp \left[-\varepsilon_{\lambda} \cdot c\left(t_{m}\right) \cdot 1\right]
$$

where $c\left(t_{m}\right)$ is the concentration in the homogeneous state. Therefore,

$$
c\left(t_{m}\right)=\frac{c_{1}(t)+c_{2}(t)+\cdots+c_{n}(t)}{n}
$$

$P\left(t_{m}\right)$ is also given by

$$
P\left(t_{m}\right)=\left.P_{0} \cdot\right|_{i=1} ^{n} \exp \left[-\varepsilon_{\lambda} \cdot c_{i}(t) \cdot 1\right]_{\mid}^{\frac{1}{n}}
$$

The next inequality ; the relation between arithmetic mean and geometric mean, is obtained by comparing $P(t)$ and $P\left(t_{m}\right)$, since each exponential term $a_{i}$ is a positive number.

$$
P(t) / P_{0}=\frac{1}{n} \sum_{i=1}^{n} a_{i} \geq\left(\prod_{i=1}^{n} a_{i}\right)^{\frac{1}{n}}=P\left(t_{m}\right) / P_{0}
$$

where In this equation, $P(t)$ and $P(t m)$ have the same value when each exponential term is equal, which means all concentrations 
where $c(t, v)$ means the mass concentration at the unit volume $v$, at time $t$, and $z(v)$ is the height in the position of the volume $v$. The concentration was standardized by the initial concentration $c_{0}$. $s(t)$ represents the variance of mass diffused. The larger the value of $\mathrm{zg}(\mathrm{t})$, the higher the micro-plume is injected, which results in well mixure in the axial direction. Likewise, when the value of $\sigma(t)$ becomes larger, the micro-plume is dispersing more and becomes well mixed.

\section{Simulation results}

First, we checked the influence on the diffusion flow of the nozzle diameter $d$, which relates to the injection velocity. Figure 5 shows a comparison of $d=10,20$, and $30 \mu \mathrm{m}$ when the flow rate is $4 \mu \mathrm{l} / \mathrm{s}$, giving mean values for injection velocity of $127 \mathrm{~mm} / \mathrm{s}(\mathrm{d}=$ $10 \mu \mathrm{m}), 32 \mathrm{~mm} / \mathrm{s}(d=20 \mu \mathrm{m})$, and $14 \mathrm{~mm} / \mathrm{s}(d=30 \mu \mathrm{m})$. All results show similar curves for both $\mathrm{z}_{\mathrm{g}}(\mathrm{t})$ and $\mathrm{s}(\mathrm{t})$ for this large velocity range. The injection flow from the nozzle was found to be a laminar jet, since the Reynolds number (taking the diameter $d$ as the representative length) is of the order of 1 . It is therefore assumed that a viscous force moderates the injection flow immediately atter it comes out the nozzle. Here, we chose $d=20 \mu \mathrm{m}$ as the diameter for the following analysis.

Next, we discuss the influence of the injection flow rate, using $q=1.0 \mu \mathrm{l} / \mathrm{s}, 4.0 \mu \mathrm{l} / \mathrm{s}$, and $8.0 \mu \mathrm{l} / \mathrm{s}$, and a total injection volume of $1 \mu \mathrm{l}$. The flow stops at $1.0 \mathrm{sec}(1.0 \mu \mathrm{l} / \mathrm{s}), 0.25 \mathrm{sec}(4 \mu \mathrm{l} / \mathrm{s})$, and $0.125 \mathrm{sec} .(8 \mu \mathrm{l} / \mathrm{s})$. Figure 6 shows the variance of $q=1.0 \mu \mathrm{l} / \mathrm{s}$ at 1.0 sec. is $34 \times 10^{2}\left(\mu l^{2}\right)$, relatively small compared to the value of $42 \times 10^{2}\left(\left.\mu\right|^{2}\right)$. A quick injection causes the mass to disperse faster. We see that convective flow accelerates the dispersion more. However, it must be noted that even when the flow rate $q=$ $1.0 \mu \mathrm{l} / \mathrm{s}$, the mixing is done quickly. Hence, we used $q=4 \mu \mathrm{l} / \mathrm{s}$ as the flow rate for the following simulation.

Figure 7 is the change of variance depending on the height (h), from $110 \mu \mathrm{m}$ to $440 \mu \mathrm{m}$. Here, the injection stops after $0.25 \mathrm{sec}$. This shows $\sigma(\mathrm{t})$ reaches a maximum around $300 \mu \mathrm{m}$.

In sum, a diameter of around $20 \mu \mathrm{m}$, and a height of $300 \mu \mathrm{m}$ is suggested, and a flow rate more than $4 \mu \mathrm{l} / \mathrm{s}$ is best, but the last depends on the performance of the pump.
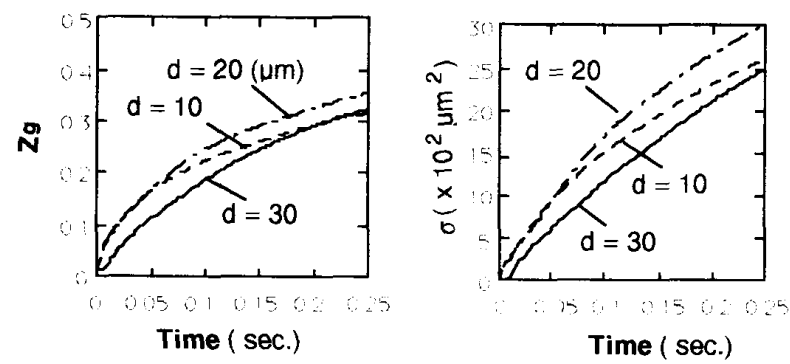

Fig. 5 Diffusion depending on Nozzle Diameter
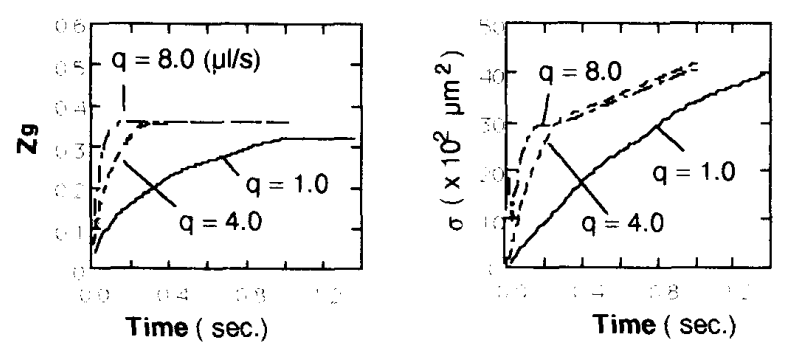

Fig. 6 Diffusion depending on Injection Flow rate

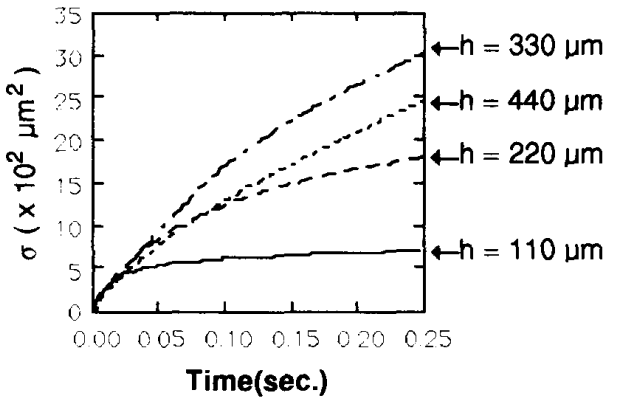

Fig.7 Diffusion depending on Height of Mixing Area

\section{FABRICATION}

The fabrication process of the mixer is simple, as shown in Fig. $8(a)$. The nozzle holes are etched using $\mathrm{KOH}$ into a 3 inch (100)Si wafer. Next, the channel for the sample liquid is etched from the other side, until holes are opened. A Pyrex glass wafer with inlet ports for the sample liquid and a cover glass with an optical reflector are anodically bonded to both sides of the Si wafer. Figure $8(b)$ illustrates the configuration of the micro mixer. This mixer has an inlet port for the sample (left side), an inlet port for the reagent (center), and a mixing area (center) which is $2.2 \mathrm{~mm} \times 2$ $\mathrm{mm} \times 330 \mu \mathrm{m}$ and has 400 micro-nozzles $(15 \mu \mathrm{m} \times 15 \mu \mathrm{m})$ on its bottom.
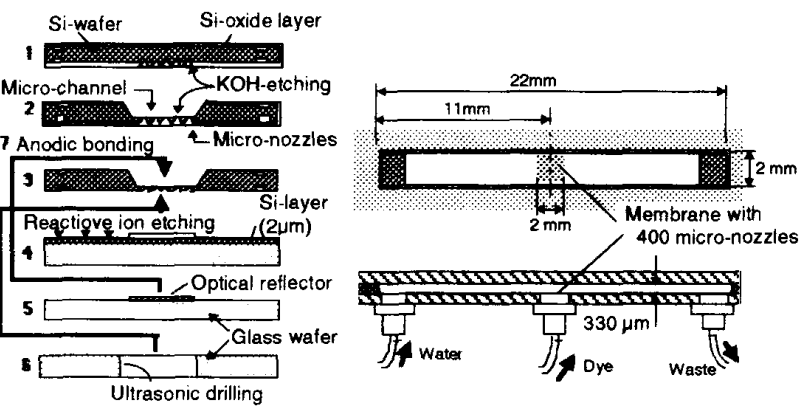

(a) Fabrication Process

(b) Configuration of Micro Mixer

Fig. 8 Fabrication of Micro Mixer

Figure 9 shows a SEM view of the nozzles fabricated by isotropic etching. Due to this simple fabrication process, the mixer can be easily integrated with the other micro-liquid handling devices on a single chip.

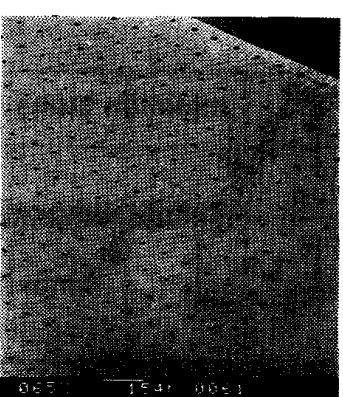

(a) 400 Mlcro-Nozzles

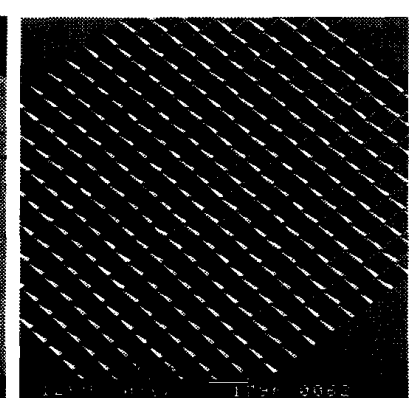

(b) Reverse side of Nozzles
Fig. 9 SEM View of 400 Micro-Nozzles Distance between nozzles : $100 \mu \mathrm{m}$ 
$c_{i}(t)$ are equal. Therefore, it is deduced that, when the output light power takes a minimum value, the concentration becomes uniform in the direction perpendicular to the light beam. On the other hand, the concentration in the beam direction also becomes uniform, since the flow from each micro nozzle is symmetrical. In this optical setup, it is possible to measure the time to complete mixing by checking the output light power absorbed.

\section{Measurement results}

This optical setup is calibrated with regard to the stability of the baseline in the blank state (no dye in the optical path) and the feasibility to work as an absorptiometer. For the stability, the change of the output power is within $1.5 \%$ of the total value for 1 $\min$. The stability is therefore enough to check the time taken for mixing.

For the feasibility, the correlation between the concentration of liquid and the output power is checked. If it works as an absorptiometer, the next relation is given from the equation (6).

$$
-\ln (P / P 0)=k \cdot c, k=\varepsilon_{\lambda} \cdot 1
$$

The mixing area is filled with dyes at several concentrations in turns. The Rhodamine concentration used in this experiment is about $10^{-5}$ wt $\%$. As depicted in Fig. 13, a linear correlation was obtained.

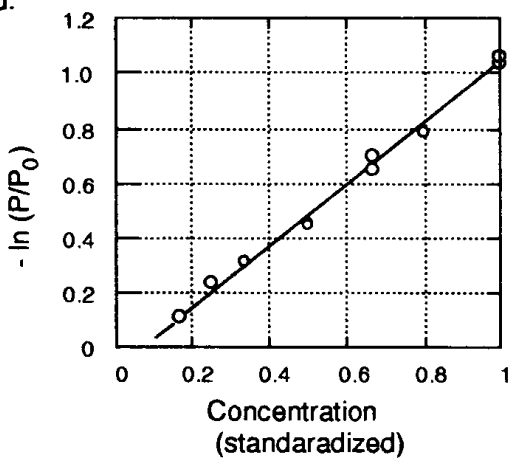

Fig. 13 Feasibility of Optical Setup

Using this setup, the time to complete mixing is investigated. Figure 14 shows the results of measurements when the injection flow rate is $0.75 \mu \mathrm{l} / \mathrm{s}$, the total volume injected is $0.5 \mu \mathrm{l}$, and the injection stops after $0.67 \mathrm{sec}$. The output signal continuously decreases after the injection stops, and an equilibrium state is reached in $1.2 \mathrm{sec}$. later. Thus, as seen in the flow visualization, this measurement reveals the mixing is completed within a few seconds.

\section{DISCUSSION}

The concept of the micro-liquid handling system was illustrated in Figure 1. The liquid has to be transported to the detector after the mixing completes. Here, we discuss the application of the the micro-mixer to this detector components with regard to the relative position in the system.

As mentioned earlier, the mixing area can be treated as a micro reaction beaker. It is feasible to set the detector in the mixing area and measure directly (Fig. 15 (a) ). This method has the advantage of making it possible to measure the early stages of mixing and reaction, and the system is simply integrated with the detector.

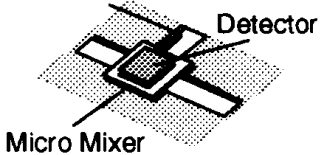

(a) Detection at Mixer

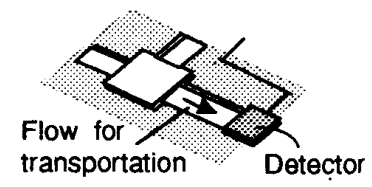

(b) Detection at Downstream

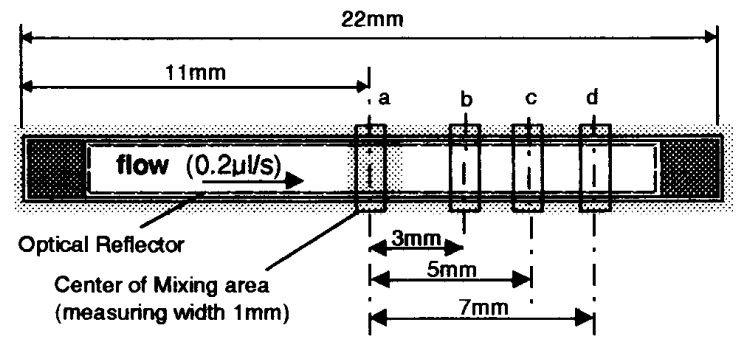

Fig. 15 Application to Detector

The setup shown in Fig.15 (b), with the detector set downstream from the mixer, has the advantages that a more complicated detector can be used, and that the number of measurements per unit time may be increased by performing mixing and detection of successive samples simultaneously. However, as the mixed fluid is conveyed to the detector location, its concentration profile is deformed by convection. We observed this deformation and then checked the feasibility of this method.

As depicted in Fig. 10, the pair of optical fibers are positioned along the flow channel with an xyz-positioner. Figure 15 illustrates the measurement positions, which are the center of the mixing area, $3 \mathrm{~mm}$ from the center, $5 \mathrm{~mm}$, and $7 \mathrm{~mm}$. The time-dependent signal from each position is shown in Figure 16 for a transportation flow rate of about $0.3 \mu \mathrm{l} / \mathrm{s}$. Thus, the deformation of concentration occurs quickly only in the first few millimeters downstream. Figure 17 gives the reproducibility of the concentration profile observed at $5 \mathrm{~mm}$ and $7 \mathrm{~mm}$ downstream. This shows that

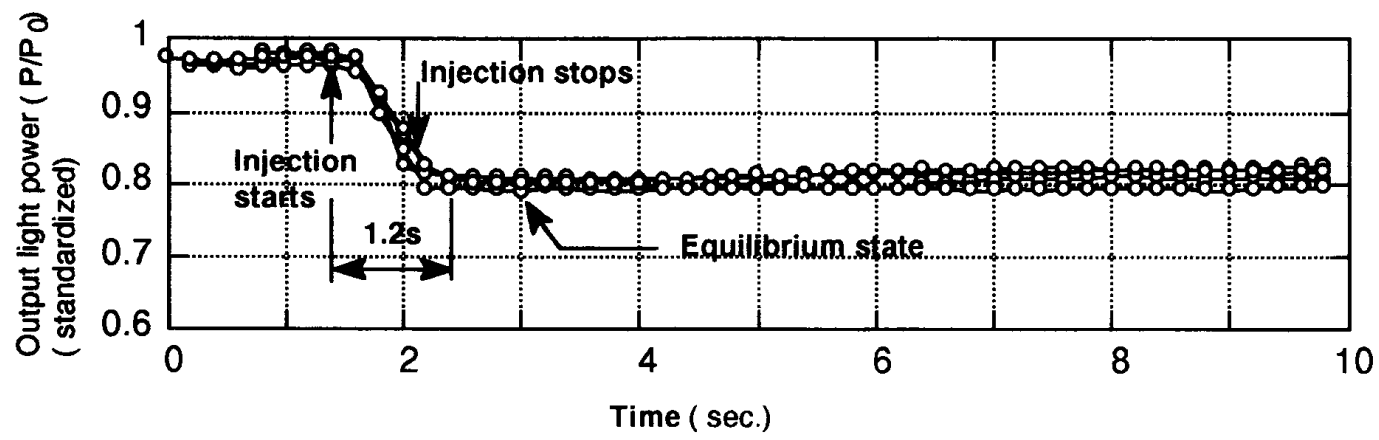

Fig. 14 Experimental Result 
the profiles are quite similar. It may be possible to use this method, even though the profile changes drastically due to the transporting flow.

Thus, both of the above methods for detection are feasible. One must be selected on the basis of usage and their advantages, and further detailed investigation, both experimental and numerical, on matching in actual systems is necessary.

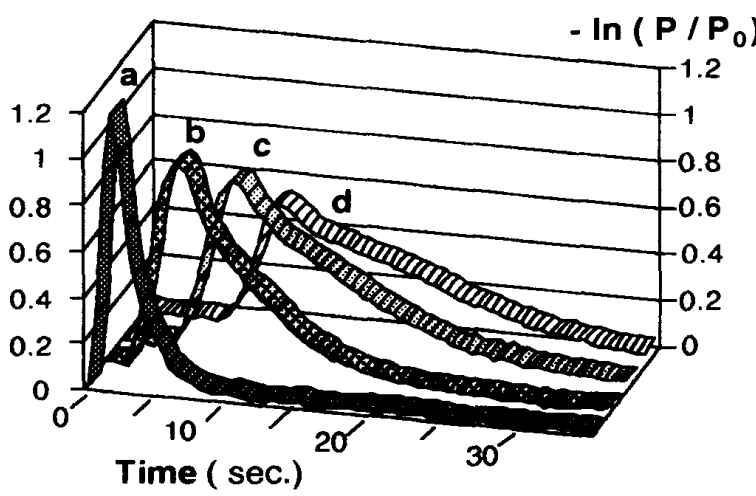

Fig. 16 Deformation of Concentration
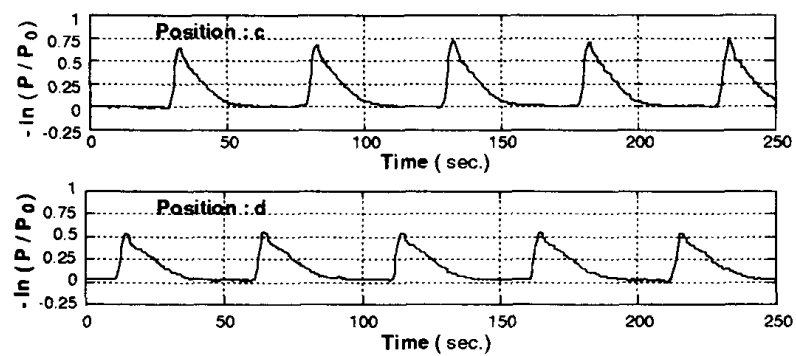

Fig. 17 Reproducibility of Concentration Profile

\section{CONCLUSION}

A new concept for micro mixing utilizing the effect of molecular diffusion is introduced and its feasibility is demonstrated numerically and experimentally. The mixing process using the micro mixer is faster by a factor of $\sim 100$ as than that of a single plume, according to the numerical simulation.

Flow visualization by microscope revealed that mixing is complete within a few seconds, and an absorptiometry using a pair of optical fibers showed that a homogeneous state of mixing is achieved in 1.2 seconds, when the total volume injected is $0.5 \mu \mathrm{l}$ and the injection flow rate is $0.75 \mu \mathrm{l} / \mathrm{s}$.

This micro mixer is applicable to micro-liquid handling systems due to its simple mixing method and fabrication process.

\section{ACKNOWLEDGEMENTS}

The author would like to thank those involved from the staff of the MESA Research Institute of the University of Twente, particularly Dr. H. Gardeniers, Dr. C. Eykel, C. van Mullen, D. Ekkelkamp, E. Berenschot, and $\mathrm{H}$. van. Vossen for their valuable cooperation, and $\mathrm{U}$. Lindberg for his useful suggestions.

\section{REFFERENCES}

[1] Frans C.M. van de Pol,"Micro Fluid Handling Systems," 5th Int. Cont. on Advanced Robotics, June 19-22, 1991, Pisa, Italy, pp.281-290, 1991

[2] F.C.M.Van de Pol, H.T.G.Van de Lintel, M. Elwenspoek and J. H. J. Fluitman, "Thermopneumatic Micropump Based on Micro-engineering Techniques," Sensors and Actuators, vol.A21-A23, pp.198-202, 1990

[3] T. Onnstein, Fukida, T., J. Ridley, and U. Bonne, "Micro Machined Silicon Microvalve," Proc. of MEMS'90, pp.95-98, 1990

[4] Esashi, M., Shoji, S., and Nakano, A., "Normally Closed Microvalve and Micropump Fabricated on a Silicon Wafer," Sensors and Actuators, 20, pp.163-169, 1990

[5] A. Manz, N. Graber, and H. M. Widmer, "Miniaturized Total Chemical Analysis Systems: A Novel Concept for Chemical Sensing," Sensors and Actuators, B1, pp.244-248, 1990

[6] Theo S. J. Lammerink, Miko Elwenspoek, and Jan H. J. Fluitman."Integrated Micro-Liquid Dosing System," Proc. of MEMS'93, 1993

[7] R. S. Brodkey, "Turblence in Mixing Operations,"Academic Press, Inc., New York, 1975

[8] E. L. Cussler, "Diffusion Mass Transfer in Fuid Systems," Cambridge Univ. Press, New York, pp.52-53, 1984

[9] E. S. Oran, and J. P. Boris, "Numerical Simulation of Reactive Flow," Elsevior, New York, 1987

[10] J. C. Sternberg,"Advances in Chromatography Vol. 2," Marcel Dekker, New York, pp.205-270, 1966

[11] Miyake, R., Onki, H., Yamazaki, I., and Yabe, R.,"Development of Micro Sheath Flow Chamber," Proc. of MEMS'91(1991), pp265-270

[12] O. H. Wheeler, A. A. Kaplan, et al,"Organic Electronic Spectral Data vol. 3," Inter Science Publisher, New York, pp1025

\section{APPENDIX}

The basic equations of this finite differential analysis are a vortex transport equation and a diffusion equation [9]. The boundary conditions for the wall are given as follows:

$$
\mathbf{u} \equiv \mathbf{0}
$$

In the outlet, we suppose the change of velocity in the r-direction is moderate enough and the flow in the axial direction is small enough as compared to that in the r-direction. For the diffusion, we suppose the change of flux is moderate enough. Threfore, we get

$$
u_{z}=0, \quad \frac{\partial u_{r}}{\partial r}=0, \frac{\partial^{2} c}{\partial r^{2}}=0
$$

In the center, since the flow and diffusion are symmetrical, the condition is given by

$$
u_{r}=0, \frac{\partial u_{z}}{\partial r}=0, \frac{\partial c}{\partial r}=0
$$

In the inlet, we suppose a Poiseuilli flow profile. 